\title{
US Physicians with Recent Experience Managing Hospitalized Older Adults: an Observational Study
}

J Gen Intern Med 35(12):3747-9

DOI: $10.1007 / \mathrm{s} 11606-020-06240-\mathrm{Z}$

(c) Society of General Internal Medicine 2020

\section{INTRODUCTION}

The number of COVID-19 cases in the USA surged from zero to 1.7 million between February and May $2020 .{ }^{1}$ With predicted hospitalization rates as high as $20 \%,{ }^{2,3}$ many hospitals ramped up staffing by deploying physicians to the front lines. ${ }^{4}$ However, little is known about the supply of physicians potentially available to manage hospitalized patients with COVID-19. Over the past 20 years, US healthcare delivery shifted from predominantly facility-based to ambulatory clinic-based services, with some physicians no longer treating hospitalized patients. As hospitals face the COVID-19 pandemic, an assessment of demographics and practice characteristics of physicians likely to be deployed could inform resource allocation. For example, given elevated risk of severe disease among older adults, ${ }^{2}$ hospitals may not want to deploy older physicians to provide direct patient care. Our objectives were to (1) describe the characteristics of physicians with recent experience managing hospitalized patients, and (2) measure regional prevalence of these physicians across hospital service areas (HSAs) ${ }^{5}$.

\section{METHODS}

We used the 2017 Provider Utilization and Payment Data, containing physician billings for Medicare fee-for-service beneficiaries, linked to the Medicare Physician Compare files, containing physician gender and year of medical school graduation.

First, we identified all 579,056 physicians who billed for > 10 evaluation and management visits. Because Medicare billing codes for hospital visits are the same for primary attending and consultants, we excluded specialties least likely to serve as the primary team for a medicine patient (dermatology, nuclear medicine, ophthalmology, psychiatry, pathology, and radiology) (16.8\%), and emergency medicine physicians (7.4\%) who are more likely to be deployed in the emergency department. We then used hospital visit (99217-99226, 99231-

Received April 28, 2020

Accepted September 11, 2020

Published online September 19, 2020
99236, 99238, 99239), critical care (99291, 99292), and ventilator management codes (94002-94005, 94660-94662) to identify physicians who treated hospitalized (or critically ill) patients. Lastly, we measured the prevalence of physicians per 100,000 residents in each HSA.

Variables were compared using the $\chi^{2}$ test. Two-sided $p$ values $<0.05$ were considered significant. Analyses were conducted using STATA 16.0 (College Station, TX). The University of Pennsylvania Institutional Review Board waived review of this study.

\section{RESULTS}

Of the 438,895 physicians in our sample, $44.8 \%$ provided hospital and $7.2 \%$ critical care visits. The majority were male (75.0\% of physicians with hospital visits and $77.2 \%$ with critical care visits) (Table 1). Ten percent (10.2\%) of physicians with hospital and $6.6 \%$ of physicians with critical care visits graduated $41+$ years ago. Many performed 50 or fewer visits $(17.3 \%$ for hospital visits and $43.2 \%$ for critical care visits).

Across the 3360 HSAs, the median number of physicians who treated hospitalized patients was 35.8 per 100,000 residents (inter-quartile range [IQR] 18.9 to 58.3). This population-based prevalence varied 7 -fold between the $25 \%$ of HSAs with the highest and lowest prevalence. The median number of physicians who provided critical care was 3.4 per 100,000 residents (IQR 0 to 8.8 ). Forty percent (40.6\%) of HSAs had no physicians who billed for $>10$ critical care visits (Fig. 1).

\section{DISCUSSION}

More than half of all physicians who billed Medicare in 2017 provided fewer than 11 hospital visits. Applying additional criteria such as critical care experience, risk factors for severe COVID-19 (age $>65$ based on years of experience), and minimum visit volume further reduced the estimates of physicians with recent experience potentially available to treat hospitalized patients.

This study has limitations. First, we did not assess care not covered by fee-for-service Medicare. Although very few physicians opt out of Medicare, ${ }^{6}$ some may accept non-traditional Medicare products (such as Medicare Advantage) in lieu of traditional Medicare. While Medicare Advantage plans 
Table 1 Characteristics of Physicians Who Treated Hospitalized Patients Overall and Those Who Provided Critical Care in 2017

\begin{tabular}{|c|c|c|c|c|}
\hline \multirow[b]{2}{*}{ Characteristics* } & \multicolumn{2}{|c|}{$\begin{array}{l}\text { Physicians who treated hospitalized } \\
\text { patients }(N=196,788)\end{array}$} & \multicolumn{2}{|c|}{$\begin{array}{l}\text { Physicians who treated critical } \\
\text { care patients }(N=31,647)\end{array}$} \\
\hline & $n$ & $\%$ & $n$ & $\%$ \\
\hline \multicolumn{5}{|l|}{ Gender } \\
\hline Male & 147,496 & 75.0 & 24,429 & 77.2 \\
\hline Female & 49,292 & 25.0 & 7218 & 22.8 \\
\hline \multicolumn{5}{|c|}{ Years from medical school graduation ${ }^{\dagger}$} \\
\hline $0-10$ & 19,675 & 10.1 & 3202 & 10.3 \\
\hline $11-20$ & 63,817 & 32.8 & 12,162 & 39.1 \\
\hline $21-30$ & 50,267 & 25.8 & 8161 & 26.2 \\
\hline $31-40$ & 40,957 & 21.0 & 5542 & 17.8 \\
\hline $41+$ & 19,902 & 10.2 & 2051 & 6.6 \\
\hline \multicolumn{5}{|l|}{ By volume of visits } \\
\hline $11-50$ visits & 34,132 & 17.3 & 13,669 & 43.2 \\
\hline $51-250$ visits & 66,392 & 33.7 & 13,407 & 42.4 \\
\hline $250-500$ visits & 35,425 & 18.0 & 3360 & 10.6 \\
\hline $501-1000$ visits & 32,000 & 16.3 & 1058 & 3.3 \\
\hline $1001+$ & 28,839 & 14.7 & 153 & 0.5 \\
\hline \multicolumn{5}{|l|}{ Top 5 specialties $^{\S}$} \\
\hline Internal medicine & 51,871 & 26.4 & 9822 & 31.0 \\
\hline Family practice & 17,054 & 8.7 & 2212 & 7.0 \\
\hline Cardiology & 16,501 & 8.4 & 2174 & 6.9 \\
\hline General surgery & 12,884 & 6.7 & 1708 & 5.4 \\
\hline Pulmonary/critical care & 11,673 & 5.9 & 9484 & 30.0 \\
\hline Other & 86,805 & 44.1 & 6247 & 19.7 \\
\hline
\end{tabular}

*p value $<0.001$ for all comparisons across categories of physician characteristics

†Year of graduation from medical school was available for 194,618/196,788 physicians (1.1\% missing)

f "Other" category includes anesthesiology, medical and surgical subspecialists, medicine/pediatrics, orthopedic surgery, neurosurgery, neurology, obstetrics and gynecology, otolaryngology, preventative medicine and rehabilitation, and urology

${ }^{\S}$ Percentages do not add up to 100 due to rounding

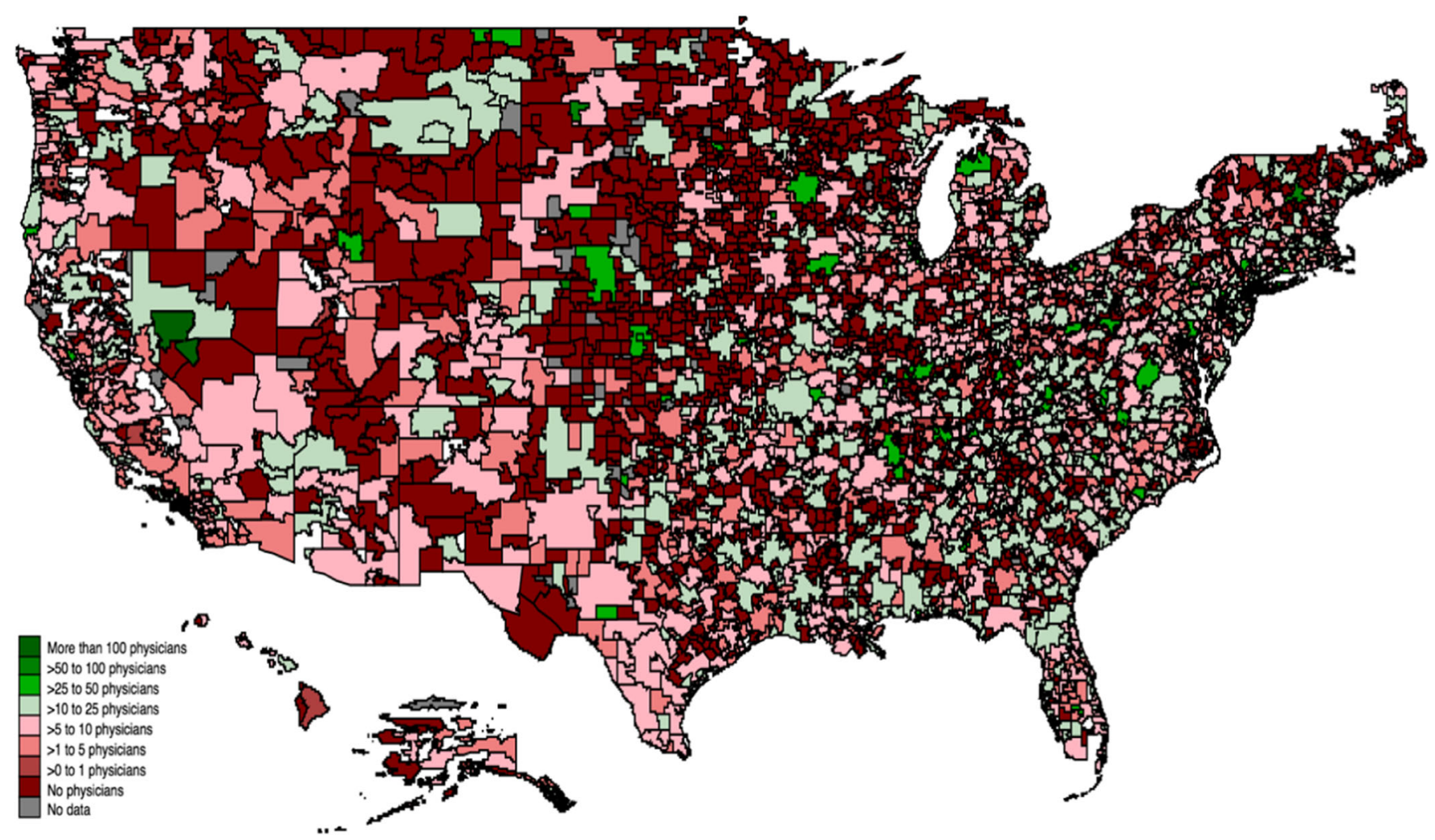

Figure. 1 Prevalence of physicians who treated hospitalized Medicare beneficiaries requiring critical care per 100,000 hospital service area residents in 2017. The figure shows the geographic distribution of physicians who billed Medicare for critical care services in 2017 per 100,000 residents in each hospital service area. 
generally restrict participants' choice of physicians using narrower networks of participating providers compared to traditional Medicare, it is possible that our approach may slightly undercount the number of available physicians. Second, we did not account for willingness of physicians to change their practice during a pandemic. Third, we did not count trainees who provide a crucial component of hospital care.

Our findings anticipate a shortage of physicians with recent experience managing hospitalized patients with critical care needs during a pandemic, particularly in some areas of the country. Planning efforts should include strategies to mitigate this shortage, including the use of telemedicine, retraining modules for physicians without recent acute hospital care experience, and ensuring that the limited healthcare workforce is protected from COVID-19 with adequate personal protective equipment.

Kira L. Ryskina, MD, MSHP ${ }^{1,2}$

Anjali Bhatla, $B A^{3}$

Rachel M. Werner, $\mathrm{MD}, \mathrm{PhD}^{1,2,4}$

${ }^{1}$ Division of General Internal Medicine, Perelman School of Medicine of the University of Pennsylvania,

Philadelphia, PA, USA

${ }^{2}$ Leonard Davis Institute of Health Economics, University of Pennsylvania,

Philadelphia, PA, USA

${ }^{3}$ Perelman School of Medicine of the University of Pennsylvania,

Philadelphia, PA, USA

${ }^{4}$ Crescenz VA Medical Center, Philadelphia, PA, USA

Corresponding Author: Kira L. Ryskina, MD, MSHP; Division of General Internal Medicine, Perelman School of Medicine of the University of Pennsylvania, Philadelphia, PA, USA (e-mail: ryskina@pennmedicine.upenn.edu).
Funding Dr. Ryskina's work on this study was supported in part by the NIA Career Development Award (K08AG052572). Dr. Werner was supported in part by K24-AG047908 from the NIA.

Data Availability Dr. Ryskina had full access to all the data in the study and takes responsibility for the integrity of the data and the accuracy of the data analysis. Dr. Ryskina conducted analyses for this study.

\section{Compliance with Ethical Standards:}

Disclaimer: The NIH had no role in the design and conduct of the study; collection, management, analysis, and interpretation of the data; preparation, review, or approval of the manuscript; and decision to submit the manuscript for publication.

Conflict of Interest: The authors declare that they do not have a conflict of interest.

\section{REFERENCES}

1. Centers for Disease Control and Prevention. Coronovirus Disease 2019. Cases in the U.S. Accessed on May 26, 2020 at https://www.cdc.gov/ coronavirus/2019-ncov/cases-updates/cases-in-us.html

2. Guan WJ, Ni ZY, Hu Y, et al. Clinical characteristics of Coronavirus disease 2019 in China. N Engl J Med 2020. DOI: https://doi.org/10. 1056/NEJMoa2002032

3. IHME COVID-19 Health Service Utilization Forecasting Team. Forecasting COVID-19 impact on hospital bed-days, ICU-days, ventilator days and deaths by US state in the next 4 months. MedRxiv. March 26, 2020; Accessed on April 5, 2020 at http://www.healthdata.org/research-article/ forecasting-covid-19-impact-hospital-bed-days-icu-days-ventilator-daysand-deaths

4. Ramachandran S, Palazzolo J, West MG, Evans M. Coronavirus cases strain New York City hospitals: 'We're getting pounded'. Wall Street J. 2020.

5. Dartmouth Atlas Group. Hospital Referral Region to Zip Code Crosswalk. Robert Wood Johnson Foundation and the Dartmouth Clinical and Translational Science Institute. Accessed on April 1, 2020 at https:// atlasdata.dartmouth.edu/static/supp_research_data

6. Boccuti C, Swoope C, Damico A, Neuman T. Medicare patients' access to physicians: a synthesis of the evidence. Menlo Park: The Kaiser Family Foundation; 2013. Accessed on April 5, 2020 at https://www.kff.org/ medicare/issue-brief/medicare-patients-access-to-physicians-a-synthesis-of-the-evidence/.

Publisher's Note: Springer Nature remains neutral with regard to jurisdictional claims in published maps and institutional affiliations. 